\title{
MEASUREMENT OF HEPATIC BLOOD FLOW BY INDICATOR DILUTION TECHNIQUES ${ }^{1,2}$
}

\author{
By STANLEY REICHMAN, WILLIAM D. DAVIS, JOHN P. STORAASLI, AND \\ RICHARD GORLIN
}

\author{
(From the Cardiopulmonary Laboratory, Gastroenterology Service and Radioisotope Laboratory, \\ United States Naval Hospital, Portsmouth, Va.; the Medical Clinics, Peter Bent \\ Brigham Hospital, Boston, Mass.; the Medical Service, Hospital for Joint \\ Diseases, N.Y., N.Y.; and the Gastroenterology Service, Ochsner \\ Clinic, New Orleans, La.)
}

(Submitted for publication January 2, 1958; accepted August 25, 1958)

This report is concerned with the development and application of a new technique for measurement of hepatic blood flow and portal circulation time.

The methods by which a patient with clinical liver disease can be studied have been generally limited to tests of hepatic function and tissue biopsy. Recently, however, transcutaneous splenic punctures have been employed not only for visualization of the portal circulation by radiographic techniques (splenoportography) (1-3) but also as a means of determining the level of portal venous pressure (4-7). Anatomical studies have shown that the splenic pulp communicates directly with the portal venous system (8). Similarly, radiopaque material injected into the splenic pulp is rapidly transported through the portal circulation within seconds following its instillation. Because of this direct communication, intrasplenic pulp pressure is a direct reflection of portal venous pressure. The latter has been confirmed by animal studies (4), at surgery in humans, by direct needling of the portal triads during liver biopsy and by hepatic vein wedge pressure catheterization studies (9).

The interrelationship of the splenic and portal circulations suggested that portal circulation time might be measured by injection of a tracer material into the spleen and observation of its course through the portal system. In addition, measure-

\footnotetext{
1 Supported in part by grants from the United States Public Health Service, National Heart Institute (H-2637), the Massachusetts Heart Association, and the Howard Hughes Medical Institute, Miami, Fla.

2 Presented at the National Meeting, American Federation for Clinical Research, Atlantic City, N. J., April, 1957.
}

ment of blood flow through the hepatic vascular bed might be calculated by application of the Stewart-Hamilton indicator dilution method (10). This method is based on the hydraulic principle that the flow within a moving stream may be determined by its ability to dilute a known amount of indicator measured with respect to time.

The requirements for application of the indicator dilution method to the portal circulatory system in man would appear to be :

1. It must be possible to inject a known amount of indicator substance which then remains entirely within the vascular system.

2. Once injected, all of the indicator must traverse the liver, i.e., there must be no shunt of injected indicator prior to the intrahepatic circulation.

3. The indicator must mix with the dual blood supply of the liver prior to the site of final sampling.

4. It must be possible to carry out uniform continuous or rapid intermittent sampling of the flow of blood as it exits from the area of complete mixing through to the first recirculation.

It will be the purpose of this report to demonstrate that under certain conditions these abovementioned requirements may be met and, therefore, blood flow and circulation times through the liver may be measured by a dilution technique.

\section{MATERIAL AND METHODS}

Studies were carried out in 43 subjects : 14 were normal, 16 had varying degrees of cirrhosis, 9 had acute hepatitis and 4 had hemolytic anemia. Intrasplenic puncture with injection of radioactive iodinated serum albumin was carried out in all subjects; direct recording of isotope dilution within the liver via an externally placed scintillation probe counter was performed in 39 ; 
continuous sampling of isotope dilution in hepatic venous blood was measured in 13; and residual splenic area radioactivity checked in 12 patients by a counter placed over the left upper quadrant.

Venous catheterization was performed in the usual manner. It was generally preferable to use a vein of the right arm to permit easier access to the site of the splenic puncture. As large a catheter as was tolerated was inserted (usually No. 8F) and advanced deep into a hepatic vein. Almost invariably, a branch of the right, and only rarely of the left, hepatic vein was successfully entered. Hence our observations were confined to sampling from the right hepatic lobe. A brachial artery was then cannulated with a No. 18 thin-wall indwelling Riley needle.

After hepatic venous and systemic arterial blood samples were drawn as blanks for subsequent indicator studies, splenic puncture was carried out. ${ }^{3}$ With the patient in the recumbent position, the site of maximum splenic dullness is percussed in the left mid- and posterior axillary lines. The posterior axillary line in the ninth or tenth intercostal space has been the most successful site for injection even in cases with splenomegaly.

The skin, subcutaneous tissues and parietal peritoneum are anesthetized with 1 per cent procaine using a 2 inch No. 26 needle. Next, a 3 inch No. 22 flexible spinal needle is passed perpendicularly into the splenic pulp. Prior to injection blood should be aspirated from the needle. When the needle was located in the splenic pulp and blood had been withdrawn, the entrance (procaine) syringe was disconnected and a tuberculin syringe containing $1 \mathrm{ml}$. (15 to $30 \mu \mathrm{c}$.) of radioactive iodinated serum albumin attached in its place. At a given signal, the small volume of indicator was rapidly injected (within one second) and sampling from the hepatic system begun.

Two techniques of indicator sampling have been employed to measure liver blood flow:

1. Continuous withdrawal of hepatic venous blood.4 Blood from the hepatic venous catheter was led past a lead-shielded scintillation probe via polyethylene tubing. The probe was connected in series to a decade scaler and a count rate computer. ${ }^{5}$ Withdrawal of blood from the

3 All patients to be studied should have normal prothrombin times, platelet counts and absence of other abnormal bleeding tendencies. Infrequently, some degree of discomfort may be experienced particularly in anxious patients who are unable to cooperate well. We have seldom used any prior medication, but have used analgesia as needed. Almost all of our 200 patients were essentially asymptomatic within an hour after the procedure except for some localized discomfort. In two patients there were signs of mild peritoneal irritation which persisted for 24 to 48 hours.

4 The initial studies were done using intermittent sampling via a multiple syringe manifold, but this was abandoned in favor of continuous sampling.

5 Beckman Instruments, Berkeley, Cal. The computer is a two-channel device which stores counts at prede-

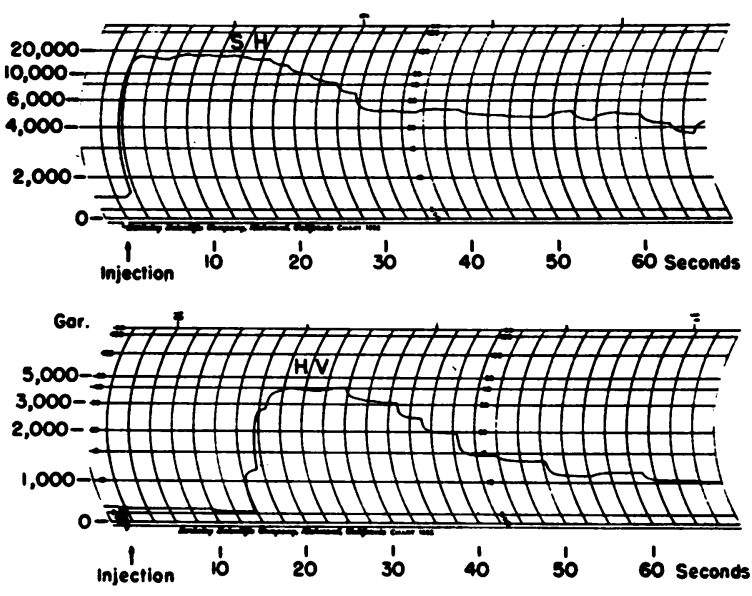

Fig. 1. Simultaneous Continuously Recorded Suprahepatic and Hepatic Venous Isotope Dilution Curves

Two seconds after injection RISA "appears" by surface counting as the material enters the liver and $15 \mathrm{sec}$ onds later at the end of the catheter placed in the hepatic vein.

distal end of the tubing was begun prior to actual injection of the radioactive indicator and maintained at a steady rate by manual traction during study. Because of the inherent resistance within the catheter, it was relatively easy to maintain a steady rate of flow. The rate of flow in $\mathrm{ml}$. per second was timed carefully by another observer (average, $0.6 \mathrm{ml}$. per second). The change in concentration of radioactivity as a function of time during the primary circulation was recorded on a semilogarithmic graphic ammeter ${ }^{6}$ which received the count rate computer output (11) (Figure 1). The final 10 minute equilibrium of circulating isotope was measured in the direct recording system and then a $1 \mathrm{ml}$. blood sample counted directly in a well counter.

2. External suprahepatic surface counting. A 1 inch scintillation probe counter with a 2.5 inch collimation was placed over the right anterior costal margin at about the midclavicular line and angled slightly laterally to view a portion of the right lobe of the liver. This probe was similarly wired to a decade scaler and count rate computer ${ }^{5}$ with counts registered directly on a semilogarithmic graphic recorder.6 ${ }^{6}$ Background counts per minute were recorded over the liver. Following splenic injection, a curve of dilution was inscribed (Figure 1). The probe was left exactly in the same place over the liver; final dilution of isotope was measured at 10 minutes in the same vascular pool sampled by the probe during dilution. Finally radioactivity was measured over the subapical re-

termined rates and can respond as often as every second (11).

'Esterline Angus Co., Indianapolis, Ind., or Varian (G-11 Recorder), Palo Alto, Cal. 
gion of the right chest ${ }^{7}$; this value was subtracted from the final dilution to correct for isotope equilibrated in nonhepatic vascular tissues (skin, bone, muscle and so forth) but "seen" by the counter after body equilibration.

Fractional two second sampling was performed from the brachial artery at the same time as hepatic venous sampling. Immediately after the flow, hepatic vein and systemic arterial samples were drawn for gasometric analysis. Blood was drawn at 10 minutes as a measure of final indicator dilution to calculate blood volume obtained following intrasplenic injection. A blood volume was also measured following a separate intravenous injection of iodinated serum albumin before the hepatic flow study was carried out. Usually the indicator was injected via the catheter in the hepatic vein and fraction collection carried out from the brachial artery in order to measure hepatic-systemic arterial circulation time and cardiac output, as well as blood volume. Radioactive blood samples were analyzed in a well counter and the values recorded as counts per minute $(\mathrm{cpm})$ per $\mathrm{ml}$.

Liver blood flow was also estimated by the bromsulfalein method (12) in six patients.

In four patients undergoing splenectomy, intrasplenic sequestration of indicator was tested by direct examination of the spleen following its removal. Immediately prior to surgical removal of the spleen, $1 \mathrm{ml}$. containing $50 \mu \mathrm{c}$. of radioactive iodinated serum albumin was injected directly into the splenic pulp. The splenic vein was clamped within 5 to 45 seconds after this injection. The excised spleen was then assayed for radioactivity and compared to a known standard containing an amount of radioactivity equivalent to that injected into the spleen.

\section{OBSERVATIONS}

\section{Special studies relating to applicability of} the indicator dilution principle

A. Determination of volume of indicator injectate. 1. Splenic removal. When successful injection was performed as indicated by the fact that splenic pulp blood could be withdrawn both before and after injection, there was a rise in suprasplenic radioactivity followed by an immediate fall to levels of between 5 and 10 per cent of the total dose. This was corroborated by the findings in four patients in whom the splenic vein was clamped following injection into the

7 The counter probe "sees" isotopes in other nonhepatic tissue as well as in liver. Ideal control for nonhepatic background would best be obtained from the same area over the left costal margin. This was not practical because of possible radiation from the site of splenic injection. It was found empirically, however, that the right anterior subapical region of the chest had a radioactivity similar to the left costal margin.
TABLE I

Intrasplenic radioactive residue as measured in the isolated spleen following injection and venous ligation

\begin{tabular}{ccc}
\hline \hline Case & $\begin{array}{c}\text { Time of ligation } \\
\text { after injection } \\
\text { of RISA } \AA^{*}\end{array}$ & $\begin{array}{c}\text { \% Activity/ } \\
\text { total dose } \\
\text { given }\end{array}$ \\
\hline no. & sec. & \\
1 & 5.5 & 12 \\
2 & 10 & 10 \\
3 & 10.5 & 10 \\
4 & 45 & 3
\end{tabular}

* Radioactive iodinated serum albumin (Abbot Laboratories).

pulp at laparotomy. The 10 to 12 per cent splenic residuum at 10 seconds may have been due to reduced splanchnic flow occurring during surgery (Table I). That the tracer bolus left the spleen immediately and uniformly was demonstrated by its arrival at the liver in less than two seconds and the initial steep descent of the primary dilution curve. In approximately 20 per cent of the patients part of the injection was either extrasplenic or apparently did not leave the splenic pulp. This was demonstrated by residual radioactivity recorded over the splenic quadrant (Figure 2). This radioactivity remained static for hours following injection, with no migration into the vascular system, suggesting extrasplenic nonvascular injection.

$$
\text { R.R. }
$$

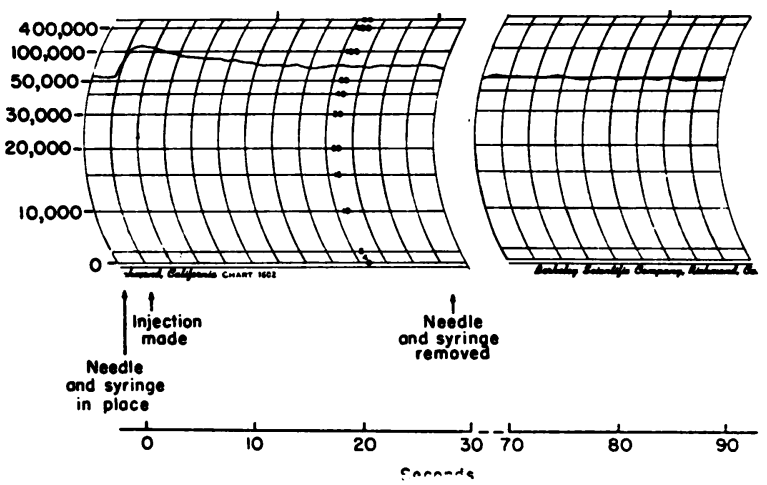

Fig. 2. Suprasplenic Activity following Poor IntraSPLENIC INJECTION

The probe was placed over the splenic area. Note that there was a high level of radioactivity over the spleen, due initially to the albumin-containing syringe. When, however, this was removed, radioactivity persisted over the splenic area as long as recorded (in this case, up to 90 seconds). 
Nevertheless, a satisfactory hepatic dilution curve could still be written from that amount of injected material which did leave the splenic pulp unless the curve was too flat in relation to the gain in activity of the final dilution sample (Figure 3 ).

2. Calculation of total effective splenic intravascular injection. Because of the frequent problem of partial extrasplenic loss, or subcapsular sequestration of indicator, it was necessary to determine "total effective intravascular injection." As described elsewhere $(11,13)$, equilibration dilution of isotope 10 minutes following intrasplenic injection was determined graphically in cpm per ml. for each direct recording system and after appropriate background correction, multiplied by a whole blood volume determined at another time. This figure gave the effective intravascular injectate as "seen" by each counting system $(11,13)$. The measurement of whole blood volume was determined by standard techniques wherein an accurate dose of indicator could be delivered intravenously and its dilution measured at the end of 10 minutes.

The accuracy of this method depends first upon

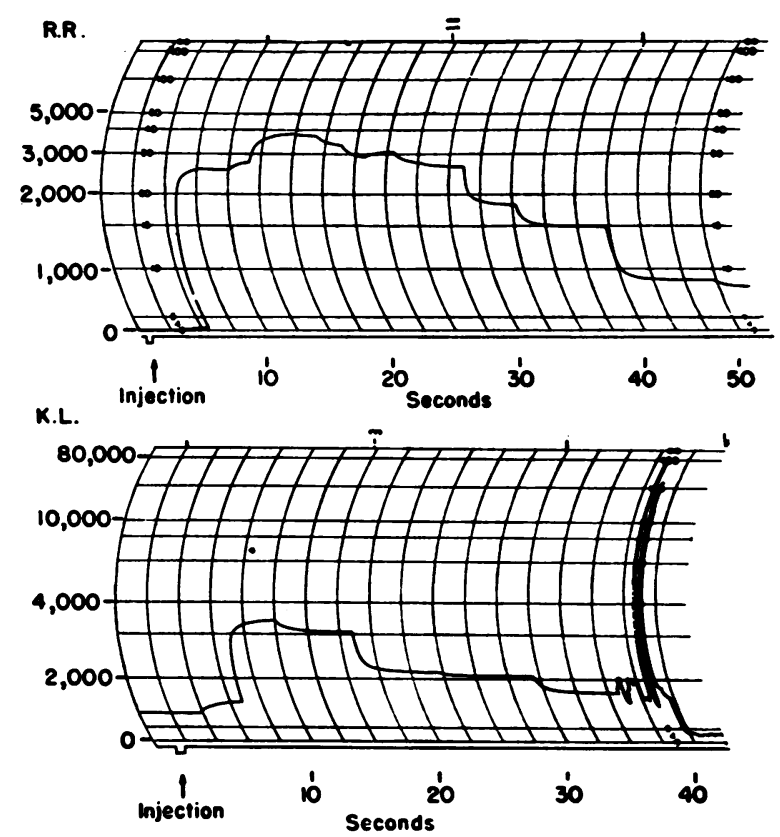

Fig. 3. Hepatic Dilution Curve following Poor INTRASPLENIC INJECTION

On the upper graph is seen trailing of indicator giving two rising peaks to the curve. the accuracy of the determination of two blood volumes and, secondly, on the nature of the left upper quadrant, presumably nonvascular extrasplenic radioactivity. The error for a single blood volume is \pm 5.4 per cent due to blood volume determination alone. Similarly, the error in the graphic recording of $\mathrm{cpm}$ per $\mathrm{ml}$. is estimated to be of the order of \pm 5 per cent. Therefore, the total probable error due to radioactive mensuration is about \pm 8 per cent.

Delayed entry of isotope (trailing in the curve) resulted only from a slow, uneven injection into the spleen. Any level of radioactivity which could be counted over the left upper quadrant after injection appeared stable without decline for hours (Figures 2 and 3 ). The method of calculation of dose from previously determined blood volume (11, 13) obviated not only errors of total intrasplenic injection but also errors in manipulation of the syringe and needle at the site of splenic puncture.

3. Lack of hepatic removal of indicator substance. $a$. With technically successful intrasplenic injection of indicator, blood volume calculated from total intrasplenic injectate equalled that obtained using intravenous injection. From this it would appear that if the liver removes any serum albumin following intrasplenic injection, it is essentially equal to that removed following intravenous injection, and this has recently been shown to be negligible (14). $b$. Suprahepatic scintillation counting curves showed a contour and regression slope similar to that of the posthepatic dilution curves (Figure 1). It would seem unlikely that the posthepatic curve would be similar to the suprahepatic curve if the liver sequestered indicator material.

$B$. Prehepatic shunts prior to sinusoidal dilution. In two of the patients with cirrhosis and varices, radioactive iodinated serum albumin was found to arrive prematurely in the brachial artery (Table V). As this did not always occur, other clinical evidence for varices served as warning that all of the indicator might not traverse the liver, and hepatic flows so estimated would be falsely high.

C. Sampling of blood stream beyond the site of complete mixing. That complete mixing of the indicator takes place before or soon after entering the hepatic circulation was demonstrated by the fact that suprahepatic counting (repre- 


\section{SUPRA HEPATIC BLOOD FLOW (LOG. SCALE)}
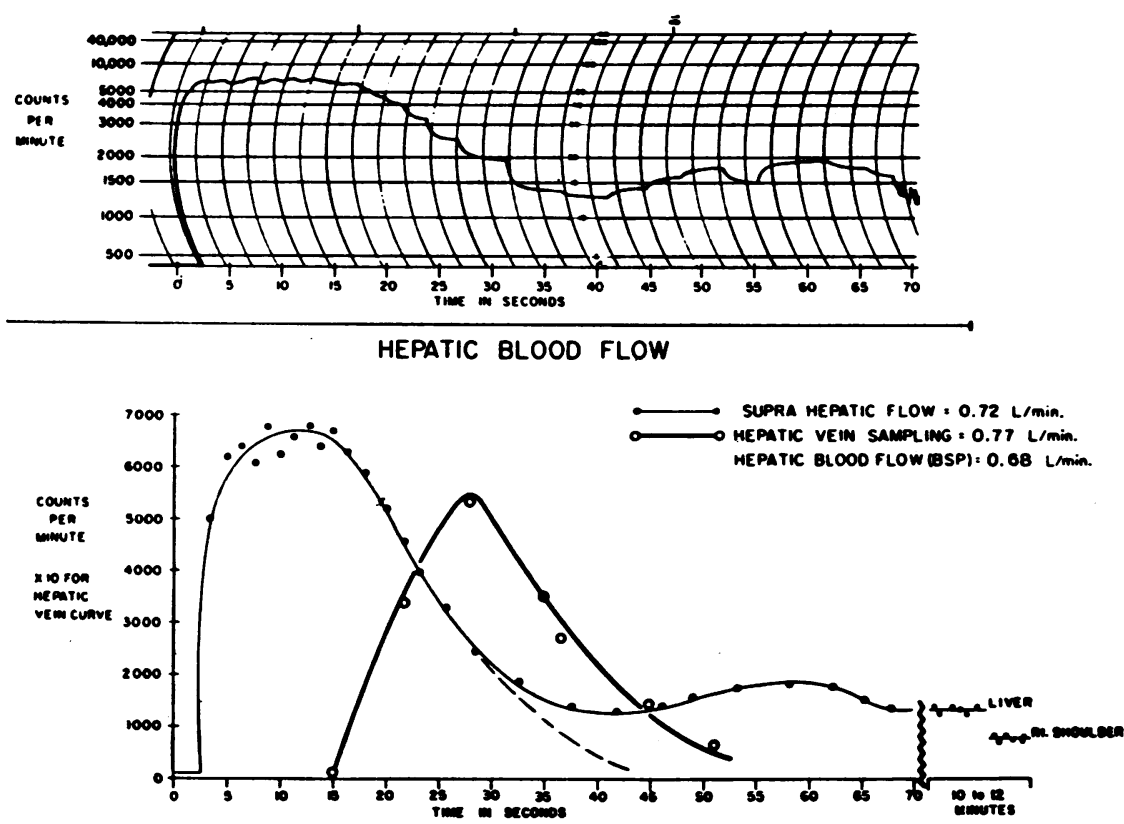

Fig. 4. Actual Curves Obtained during Simultaneous Measurements of Hepatic Flow by Suprahepatic, Hepatic Venous and Bromsulfalein Methods The phrase "suprahepatic" refers to the site of pickup of radiation.

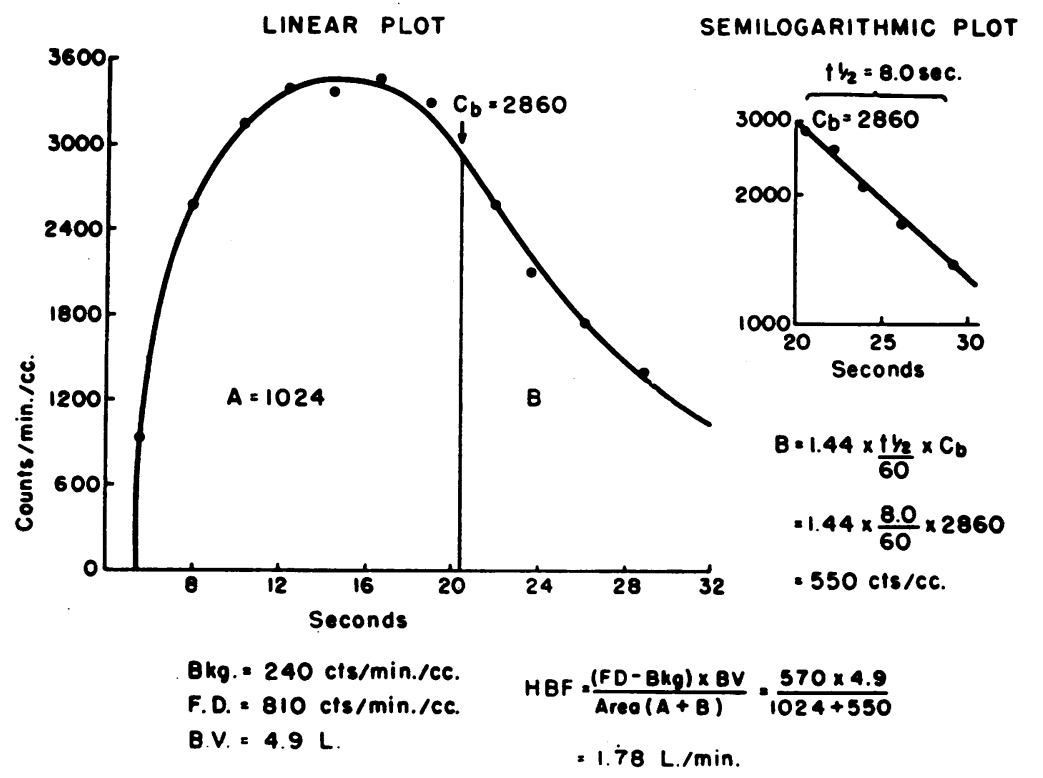

Fig. 5. Calculation of Hepatic Blood Flow

Bkg., background counts; F.D., final 10 minute dilution; B.V., whole blood volume determined independently of the hepatic flow (HBF); $A$, area under curve measured planimetrically; $B$, area under curve measured from a standard regression formula $(11) ; C_{b}$, arbitrary isotope concentration from which halftime, $t \frac{1}{2} 2$, is measured on a semilogarithmic plot. 
TABLE II

Repeated hepatic blood flow in the same patient*

\begin{tabular}{ccc}
\hline \hline & $L . /$ min. $/ M .^{2}$ & $L . / \min . / M .{ }^{2}$ \\
\hline Jer. & 0.59 & 0.66 \\
Leo & 0.86 & 0.92 \\
Cum. & 0.80 & 0.80 \\
Cap. & 0.62 & 0.62 \\
Dal. & 0.72 & 0.78
\end{tabular}

* Second flow measured after isotope equilibration of first flow measured at eight minutes.

senting intrahepatic circulation) and the hepatic venous sampling (representing posthepatic circulation) methods yielded essentially the same values for hepatic blood flow (Table III). Statistical analysis (15) as applied to these two methods indicates no significant difference in the variance between the two groups as opposed to variance within the groups, implying that the samples are homogeneous and the results of the two methods of measurement do not differ.

D. Uniform sampling of hepatic venous blood. Uniformity in sampling techniques was demonstrated first by the reproducibility of consecutive determinations of hepatic blood flow in five patients (Table II) and second, by the comparison of flows determined by three separate simultaneous methods (Table IV and Figure 4).

\section{Calculations}

A typical calculation of hepatic flow following hepatic venous sampling is shown in Figure 5. In brief, injectate is derived as the product of

TABLE III

Hepatic blood flow as measured by two dilution methods

\begin{tabular}{lcc}
\hline \hline & $\begin{array}{c}\text { Supra- } \\
\text { hepatic }\end{array}$ & $\begin{array}{c}\text { Hepatic } \\
\text { venous }\end{array}$ \\
\hline Leo & L./min./M.2 & L./min./M.2 \\
Cum & 0.86 & 0.99 \\
Cap & 0.80 & 0.89 \\
Ben & 0.62 & 0.42 \\
San & 0.70 & 0.75 \\
Gar & 0.72 & 0.77 \\
Ril & 0.71 & 0.86 \\
Cru & 0.49 & 0.64 \\
Bra & 0.57 & 0.44 \\
Col & 0.82 & 0.85 \\
But & 0.67 & 0.81 \\
McC & 0.65 & 0.68 \\
Whi & 0.60 & 0.65 \\
Average & 0.69 & 0.69 \\
& 0.72 & 0.77 \\
\hline
\end{tabular}

TABLE IV

Comparison of hepatic blood flow as measured by dilution and bromsulfalein methods

\begin{tabular}{lccc}
\hline \hline & $\begin{array}{c}\text { Supra- } \\
\text { hepatic }\end{array}$ & $\begin{array}{c}\text { Hepatic } \\
\text { venous }\end{array}$ & $\begin{array}{c}\text { Brom- } \\
\text { sulfalein }\end{array}$ \\
\hline & $L . / \min / \mathbf{M .}^{2}$ & $L . / \min . / M .^{2}$ & $L . / \operatorname{min.} / M .^{2}$ \\
Cum & 0.80 & 0.89 & 0.99 \\
San & 0.72 & 0.77 & 0.68 \\
Num & 0.57 & & 0.58 \\
Cru & 0.57 & 0.44 & 0.67 \\
Whi & 0.69 & 0.69 & 0.66 \\
Cri & 0.60 & & 0.80
\end{tabular}

blood volume times 10 minute dilution minus background. The counts along the primary curve of dilution minus the background are plotted against time; the curve is drawn and its area determined by any standard mathematical method. The method presented in Figure 5 is taken from MacIntyre, Pritchard, Eckstein and Friedell (11).

When the suprahepatic counting method is used the calculation is essentially similar except that the isotope dilution recorded over the right subapical region after 10 minutes is subtracted from the final dilution recorded over the liver at 10 minutes. In this manner, nonhepatic vascular isotope counts are eliminated in estimation of final dilution of injectate in the hepatic vascular pool (Figure 4).

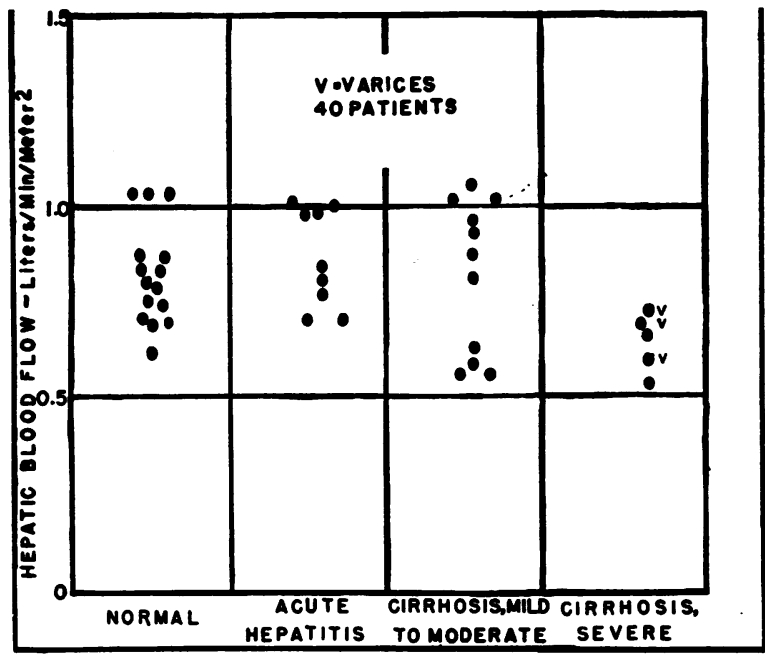

Fig. 6. The Scatter of Hepatic Flow in Normals and in Patients with Liver Disease

Note that only in the patients with severe liver disease and varices was there a marked diminution in blood flow. 
TABLE $v$

Spleno-portal circulation times

\begin{tabular}{cccc}
\hline \hline & $\begin{array}{c}\text { Spleen } \\
\text { to liver }\end{array}$ & $\begin{array}{c}\text { Liver to } \\
\text { hepatic } \\
\text { vein }\end{array}$ & $\begin{array}{c}\text { Hepatic } \\
\text { vein to } \\
\text { brachial } \\
\text { artery }\end{array}$ \\
\hline $\begin{array}{c}\text { Normal (6) } \\
\text { Average } \\
\text { Range }\end{array}$ & sec. & sec. & sec. \\
$\begin{array}{c}\text { Cirrhosis (4) } \\
\text { Average }\end{array}$ & 2.1 & 9.9 & 17.8 \\
$\begin{array}{c}\text { Range } \\
\text { Cirrhosis with } \\
\text { varices (3) } \\
\begin{array}{c}\text { Average } \\
\text { Range }\end{array}\end{array}$ & 2.4 & $4.5-13$ & $13-21$ \\
\hline
\end{tabular}

* In two patients isotope appeared at the brachial artery almost simultaneously with arrival at the liver, indicating a large prehepatic shunt was present.

\section{Results}

Results in 15 patients are shown in Tables II through IV. Blood flow as determined by the hepatic venous method yielded a value of $0.77 \mathrm{~L}$. per minute per $\mathrm{M}^{2}$, as compared to an average suprahepatic blood flow of $0.72 \mathrm{~L}$. per minute per M. ${ }^{2}$. Probable error is estimated at \pm 8 per cent. A simultaneous liver blood flow by the bromsulfalein method averaged $0.72 \mathrm{~L}$. per $\mathrm{M}^{2}{ }^{2}$ in six studies. A comparison of the three methods is shown in Figure 4.

In Figure 6 is shown a comparison of hepatic blood flows in normal patients and in patients with acute hepatitis, cirrhosis and cirrhosis with prehepatic shunts. There was no apparent change in blood flow except for a decrease in those with cirrhosis and varices.

Observations of regional circulation times are presented in Table V. Intrahepatic transit time appeared to be decreased in patients with severe cirrhosis and varices. Likewise, isotope arrived prematurely at a systemic artery relative to appearances at the hepatic vein.

\section{DISCUSSION}

In 1945 Bradley, Ingelfinger, Bradley and Curry (12) presented the first practical method of estimating hepatic blood flow in man utilizing the principle of bromsulfalein (BSP) removal by the liver. This particular method, while ingenious and a source of much valuable informa- tion, was limited by the fact that only an average rate of flow over a 40 to 60 minute period could be determined, and by the fact that in the presence of parenchymal liver disease BSP extraction may be impaired, thus decreasing the reliability of the studies. Similar objections hold true for the urea method reported by Myers (16). Hepatic vein catheterization is required for both of these techniques.

The use of tracer substances in the study of the portal circulation has recently been reported by Wheeler, Combes, Childs and Wade (17) and Tornvall and Odeblad (18). Tagged material was injected into the aorta and sampling from the hepatic vein carried out. These measurements, however, revealed only hepatic circulation times and not hepatic blood flow and were complicated by the dual supply of blood to the liver. The use of colloidal chromic phosphate and radiogold has also been described and is dependent upon the complete extraction of material in one circulation, presumably by the hepatic reticuloendothelial cells $(19,20)$. Finally, Egeli has described a portal circulation time from spleen to tongue following intrasplenic injection of decholin and calcium (21).

Evidence that the indicator dilution principle might be adapted to the splenoportal circulation was initially deduced from studies with Evans $\mathrm{T}-1824$ dye. Following its intrasplenic injection, spectrophotometric analysis of multiple hepatic vein samples indicated that a characteristic dilution curve could be obtained. However, this dye could not be recovered completely, presumably due to loss from partial extrasplenic injection and into hepatic tissues [incomplete albumin binding due to high concentration in the portal circulation (22)]. Accordingly, radioactive iodinated serum albumin was employed and an attempt made to satisfy the four criteria for application of the indicator dilution principle.

It was found that radioactive iodinated serum albumin left the spleen rapidly and passed through the liver without significant removal. In cirrhotic patients with prehepatic shunts, part of the injectate probably never entered the liver, and flows so recorded were undoubtedly falsely high or represented total splanchnic flow if mixing had occurred in the venous system. This may explain the statistically insignificant dif- 
ference in flows between normals and cirrhotics. Recognition of this possibility depended on clinical evidence of varices and premature arrival of isotope at the systemic artery whenever this occurred.

Good mixing of indicator with all sources of hepatic blood and uniform sampling of the exit stream of blood was demonstrated by comparability of duplicate flows and of flow recorded by both suprahepatic and posthepatic techniques as well as by the bromsulfalein method.

No adequate studies could be performed to compare flows recorded from right and left lobes because of the difficulty in catheterizing the left hepatic vein in man. That flow is evenly distributed is suggested by: 1) the bilobular flow of diodrast during splenoportography; 2) the studies of Cole, Krohmer, Bonte and Schatten which showed equal radioactivity over both lobes of the dog's liver no matter what preportal injection site was chosen (23); and 3) the similarity of flows derived from right hepatic vein sampling and that from externally recorded hepatic vascular pool.

The theoretical range of error of any determination was judged to be \pm 8 per cent. Normal values averaged $0.7 \mathrm{~L}$. per minute per $\mathrm{M} .{ }^{2}$, a figure approximately 20 to 25 per cent of the average resting cardiac output. The likelihood of successfully determining hepatic flow in any given patient was approximately 75 per cent.

It must be emphasized that injectate "seen" by the counter is calculated from the product of a predetermined blood volume (11) and the radioactivity in cpm per minute per $\mathrm{ml}$. in the vascular pool seen by the counter at equilibration. This means that the splenic injectate does not have to be accurately measured and that extrasplenic loss or intrasplenic sequestration will not necessarily vitiate a given flow measurement. When too much of the dose is injected outside the spleen, however, a flat curve may result with an inadequate rise in final dilution over the background count. With slow uneven injections trailing of isotope could also disturb the pattern and reliability of the curve (Figure 3 ). Finally, unsteady sampling could distort the shape of the hepatic venous curve. When any of these technical errors occurred the curves were not considered suitable for analysis.
The comparison of suprahepatic and hepatic venous techniques appears to establish the reliability of an external counting method for determination of hepatic blood flow. In institutions where venous catherization is not available, the external or suprahepatic method may be used in conjunction with intrasplenic pressure measurements for physiological studies of the portal circulation. Advantages of the method are that flow may be determined within the space of 1 minute, that the method is relatively innocuous, and that three to four measurements of flow may be determined on a given day with as little time as 10 minutes between each measurement. Because of the rapidity of the determination, this technique is suited for study of changing physiologic states such as alterations in posture, after exercise, food and the administration of drugs.

In severe cirrhosis, despite the probable shunting of some isotope past the liver, calculated blood flow was decreased. Changes were observed not only in blood flow but also in circulation times between given regions of the splenoportal hepatic circulation. For example in severe cirrhosis, intrahepatic transit time was diminished and in two individuals an hepatic vascular bypass could be detected. Such studies may be of value in the understanding of portal vein thrombosis, and the nature of the intrahepatic vascular abnormalities which occur in cirrhosis.

\section{SUMMARY}

A new technique for determining portal circulation times and hepatic blood flow is outlined. This method consists of measurement of the passage and dilution of radioactive iodinated serum albumin through the liver to the periphery following its intrasplenic injection. This pathway can be timed and followed by a scintillation counter placed externally over the liver and/or by scintillation well counting of blood collected continuously from the hepatic vein and the brachial artery.

Liver blood flow can be measured by application of the Stewart-Hamilton principle to either the hepatic vein or surface recorded radioactive dilution curve. The technique was shown to satisfy the basic requirements of an indicator dilution method except when applied to patients 
with prehepatic shunts. Methods for calibrating the total volume of effective intrasplenic injectate based on predetermined blood volume are presented.

The method was shown to be reproducible in 5 patients; similar hepatic flow values were obtained by both hepatic venous and surface scintillation counting techniques in 13 patients and compared well with the bromsulfalein method in 6 patients. Hepatic blood flow averaged 0.7 L. per minute per M. ${ }^{2}$ at rest in normal individuals. Normal spleen to liver arrival circulation time averaged 2.1 seconds; intrahepatic circulation time averaged 9.9 seconds.

\section{ACKNOWLEDGMENTS}

The authors are grateful to Hospital Corpsmen H. P. Fletcher, M. H. Smith, R. W. Guinee and D. O. Brown for their technical assistance. The secretarial aid of Mrs. Eunice Ward is appreciated.

\section{REFERENCES}

1. Rousselot, L. M., Ruzicka, F. F., and Doehner, G. A Portal venography via the portal and percutaneous splenic routes; anatomic and clinical studies. Surgery 1953, 34, 557.

2. Bahnson, H. T., Sloan, R. D., and Blalock, A Splenic-portal venography: A technique utilizing percutaneous injection of radiopaque material into the spleen. Bull. Johns Hopk. Hosp. 1953, 92, 331.

3. Child, C. G., O'Sullivan, W. D., Payne, M. A., and McClure, R. D. Portal venography. Radiology 1951, 57, 691.

4. Davis, W. D., Jr., Batson, H., and Schindel, W. The relation of intrasplenic pulp pressure to portal venous pressure, and the use of splenic explant in the study of portal venous pressure. J. Lab. clin. Med. 1954, 44, 786.

5. Reichman, S., and Davis, W. D., Jr. The splenic approach to the portal circulation. Intrasplenic and intrahepatic tissue pressure measurement in acute and convalescent hepatitis. Gastroenterology 1957, 33, 609.

6. Davis, W. D., Jr., Batson, H. M., Jr., Reichman, S., Gorlin, R., and Storaasli, J. P. Clinical applications of intrasplenic technique of portal pressure and hepatic blood flow determinations. Gastroenterology 1958, 34, 52.

7. Atkinson, M., and Sherlock, S. Intrasplenic pressure as index of portal venous pressure. Lancet 1954, 266, 1325.

8. Whipple, A. O. The problem of portal hyperten- sion in relation to the hepatosplenopathies. Ann. Surg. 1945, 122, 449.

9. Myers, J. D., and Taylor, W. J. An estimation of portal venous pressure by occlusive catheterization of an hepatic venule (abstract). J. clin. Invest. 1951, 30, 662.

10. Kinsman, J. M., Moore, J. W., and Hamilton, W. F. Studies on the circulation. I. Injection method: Physical and mathematical considerations. Amer. J. Physiol. 1929, 89, 322.

11. MacIntyre, W. J., Pritchard, W. H., Eckstein, R. W., and Friedell, $H$. L. The determination of cardiac output by a continuous recording system utilizing iodinated $\left(I^{121}\right)$ human serum albumin. I. Animal studies. Circulation 1951, 4, 552.

12. Bradley, S. E., Ingelfinger, F. J., Bradley, G. P., and Curry, J. J. The estimation of hepatic blood flow in man. J. clin. Invest. 1945, 24, 890.

13. Warren, J. V., Ed. Methods in Medical Research, Vol. 7. Chicago, Yearbook Pub., 1958.

14. Finnerty, F. A., Tuckman, J., and Buchholz, J. H. Evidence against significant extravascular distribution of Isi HSA (human serum albumin). Clin. Res. Proc. 1958, 6, 215.

15. Fisher, R. A. Statistical Methods for Research Workers. Edinburgh, Oliver \& Boyd, 1925.

16. Myers, J. D. The hepatic blood flow and splanchnic oxygen consumption of man. Their estimation from urea production or bromsulphalein excretion during catheterization of the hepatic veins. J. clin. Invest. 1947, 26, 1130.

17. Wheeler, H. O., Combes, B., Childs, A. W., and Wade, O. L. The splanchnic circulation time. Trans. Ass. Amer. Phycns 1955, 68, 177.

18. Tornvall, G., and Odeblad, E. Isotope studies on the hepatic circulation. Nord. Med. 1954, 52, 1331.

19. Dobson, E. L., and Jones, H. B. The behavior of intravenously injected particulate material: Its rate of disappearance from the blood stream as a measure of liver blood flow. Acta med. scand. 1952, 144, Supp. 273.

20. Vetter, H., Falkner, R., and Neumayr, A. The disappearance rate of colloidal radio-gold from the circulation and its application to the estimation of liver blood flow in normal and cirrhotic subjects. J. clin. Invest. 1954, 33, 1594.

21. Egeli, E. S. Transcutaneous determination of portal circulation time by decholin and calcium through the splenic route and the evaluation of the results. Forum med. (Istanbul) 1956, II, 120.

22. Hyman, C., Bernick, S., and Paldino, R. L. Histological studies on the influence of thorotrast on the intra-hepatic distribution of T-1824. Fed Proc. $1955,14,78$.

23. Cole, J. W., Krohmer, J., Bonte, F. J., and Schatten, W. An experimental study of intrahepatic distribution of portal blood. Surg. Gynec. Obstet. 1956, 102, 543 . 